\title{
Correlation Between Visfatin, Insulin Resistance (Homeostasis Model Assesment of Insulin Resistance), Inflammation (high Sensitivity C-Reactive Protein) and HDL Cholesterol Concentration in Individuals with Visceral Obesity.
}

\author{
Rini Budiyati ${ }^{1}$, Widjaja Lukito², Andi Wijaya ${ }^{1,3}$ \\ ${ }^{1}$ Prodia Clinical Laboratory, Jakarta \\ ${ }^{2}$ Faculty of Medicine University of Indonesia, Jakarta \\ ${ }^{3}$ Post Graduate Program, Hasanuddin University, Makassar
}

\section{Abstract}

\section{$\mathrm{B}$} ACKGROUND: Visfatin is a novel adipokine secreted from visceral adipose tissue and has insulinomimetic properties. Visceral obesity is a risk factor for metabolic syndrome. Insulin resistance and inflammation are linked to visceral obesity and metabolic syndrome. Dysregulation of visfatin as an adipokine could play an important role in metabolic syndrome through insulin resitance and inflammation, or lower HDL cholesterol concentration. However, this need more evidence.

METHOD: This was a crossectional study in 40 Indonesian obese men and 40 Indonesian obese women. Age: $30-60$ years in men and 50-60 years for women, from February to March 2008 in Jakarta.

RESULTS: No correlation between visfatin and hs-CRP as a marker of inflammation ( $\mathrm{r}: 0,190 ; \mathrm{P}: 0,101)$ ), or HOMA-IR as a marker of insulin resistance ( $\mathrm{r}:-0,020$; $\mathrm{P}$ : 0,246 . Suprisingly visfatin concentration is correlated with HDL Cholesterol ( $r$ : 0,416; P : 0,000

CONCLUSIONS: Visfatin plays an important role in metabolic syndrome through lipid metabolism. Positive correlation between visfatin and HDL cholesterol, was assumed that visfatin had a protective effect. Visfatin also known as as NAMPT (nicotinamide phosphoribosyltransferase) links NAD (Nicotinamide Adenine Dinucleotide) metabolism and raising of HDL Cholesterol. But the exact mechanisms need to be further studied.

KEYWORDS: Visceral Obesity, Metabolic Syndrome, NAMPT, Visfatin, HDL

\section{Introduction}

Obesity and the associated metabolic pathologies are the most common and detrimental metabolic diseases, affecting over $50 \%$ of the adult population (1) and it will increase in all over the world on the next decade affected by genetic factor, lifestyle, and overnutrition. Obesity is highly associated with inflammation, insulin resistance and risk to type 2 diabetes and cardiovascular disease.

The accumulating of adipose tissue in the abdominal visceral depot is especially correlated with insulin resistance. Several recent studies have demonstrated that adipose tissue secreted different proteins (adipocytokines), which likely have an important role in the development of obesity related complications, especially insulin resistance (2). These conditions are associated with a chronic inflammatory response characterized by abnormal cytokine production, increased acute-phase reactants, and activation of inflammatory signaling pathways (1). 
The criteria of metabolic syndrome from International Diabetes Federation, (IDF, 2005) are central obesity, and two of four criteria : triglyceride $\geq 150 \mathrm{mg} / \mathrm{dl}$, HDL (high density lipoprotein) cholesterol : male $<40 \mathrm{mg} / \mathrm{dl}$ and female $<50 \mathrm{mg} / \mathrm{dl}$, blood pressure $\geq 130 / 85 \mathrm{mmHg}$, fasting plasma glucose $\geq 100 \mathrm{mg} / \mathrm{dl}$ (3).

Adipocytokines plays important roles in energy metabolisms and when their production are not properly regulated, they could contribute to metabolic diseases. Disregulation of adipocytokines are the link between obesity, inflammation, insulin resistance and metabolic syndrome $(4,5,6)$, but the mechanisms has not fully elucidated.

Fukuhara et al., 2005 (7) isolated a newly identified adipocytokine, visfatin, that is highly enriched in the visceral fat of both humans and mice and whose expression level in plasma increases during the development of obesity. Visfatin corresponds to a protein identified previously as pre-B cell colony-enhancing factor (PBEF), a 52-kilodalton cytokine expressed in lymphocytes. Visfatin exerted insulin-mimetic effects in cultured cells and lowered plasma glucose levels in mice. Mice heterozygous for a targeted mutation in the visfatin gene had modestly higher levels of plasma glucose relative to wild-type littermates. Surprisingly, visfatin binds to and activates the insulin receptor .

The effects of visfatin on adipogenesis and glucose metabolism are of particular interest with respect to a putative role in the pathogenesis of obesity and diabetes. Some subsequent clinical studies confirmed the association of visfatin and diabetes, whereas others did not find an association. Similarly, data regarding the relationship of visfatin with parameters of glucose metabolism and insulin resistance were contradictory, and overall there was no clear effect of visfatin on metabolism. Even more controversial is the discussion the association of visfatin and obesity, with positive, negative or lack of association, all having been reported in addition, in contrast to the original publication (8)

Further study of visfatin's physiological role may lead to new insights into glucose homeostasis and/or new therapies for metabolic disorders such as diabetes $(7,9)$. The aims of this study is to find the correlation between visfatin, inflammation (hs-CRP), insulin resistance defined as HOMA-IR and HDL cholesterol in individu with visceral obesity.

\section{Methods}

The study subjects were 40 Indonesian obese men and 40 Indonesian obese women, aged $30-60$ years and $50-60$ years, respectively. Subjects with symptoms of infection(s), autoimmune diseases or any underlying acute condition as assessed by a medical interview were excluded. Writen informed consent was obtained from all subjects. This study was approved by the Ethics Commite of the Faculty of Medicine, University of Indonesia. The study subjects were recruited in Jakarta, from February until March 2008.

Heights, body weights, and waist circumferences of the study subjects were measured by the standard methods and the body mass index (BMI) was calculated according to the standard formula. Venous blood samples were collected in the morning of the day of the study after the patients had fasted overnight. The samples were examined in Prodia Clinical Laboratory. The serum concentration of insulin (mU/1) was measured by immunochemiluminescent methods (Immulite 2000). HOMA-IR was calculated according to the following formula: fasting glucose $(\mathrm{mmol} / \mathrm{L}) \mathrm{x}$ fasting insulin $(\mathrm{mU} / \mathrm{l}) / 22.5$. Cut off of insulin resistance is $>2$ ( $\mathrm{IDF}, 2002)$.

The serum visfatin $(\mathrm{mg} / \mathrm{mL})$ level was measured with an enzyme immunoassay kit (Phoenix Pharmaceuticals, Belmot , CA) . Its intra-and inter-assay coeficients of variation were $5 \%$ and $12 \%$ respectively. The minimum detection limit with this methods was $0.5 \mathrm{~g} / 1$. hs-CRP $(\mathrm{mg} / \mathrm{L})$, measured by an immunochemiluminescent assay of Immulite 2000 (Diagnostic Products Corporation).

Measurements of fasting plasma glucose (mg/ d1) was carried out with heksokinase methods (Roche Diagnostics), Triglyceride (mg/dl) with GPO-PAP methods (Roche Diagnostic) and HDL cholesterol with enzimatic homogenous methods (Daichii Diagnostics)

Analyses were performed using the SPSS for windows (version 11.5 SPSS Inc). P- values $<0.05$ were considered to be significant. 


\section{Results}

The association between visfatin levels and serum hs$\mathrm{CRP}$ levels as determined by simple regression analysis is shown in Fig. 1. The association between visfatin levels and HOMA-IR is shown in Fig 2. There is no correlation between visfatin and hs- CRP ( $\mathrm{r}: 0,190 ; \mathrm{P}: 0,101)$ or HOMA-IR (r:-0,020; P : 0,246). Suprisingly visfatin concentration is correlated with HDL Cholesterol ( $\mathrm{r}$ : $\left.0,416^{* * ; P}: 0,000\right)$ shown in Fig. 3.

Table 1. Characteristics of the study subjects.

\begin{tabular}{|c|c|}
\hline Variable & Mean \pm SD \\
\hline Number of patients $(n)$ & 77 \\
\hline \multicolumn{2}{|l|}{ Age (Years ) $46 \pm 8$} \\
\hline \multicolumn{2}{|l|}{ Genders } \\
\hline Male $(\%)$ & $40(52 \%)$ \\
\hline Female (\%) & $37(48 \%)$ \\
\hline \multicolumn{2}{|l|}{ Blood Pressure } \\
\hline Systole (mmHg) & $119,7 \pm 15,8$ \\
\hline Dyastole $(\mathrm{mmHg})$ & $80,76 \pm 9,8$ \\
\hline Body Mass Index (Kg/m2) & $27 \pm 6$ \\
\hline Waist Circumference $(\mathrm{cm})$ & $95,9 \pm 9,1$ \\
\hline Fasting Plasma Glucose $(\mathrm{cm})$ & $88,47 \pm 11,41$ \\
\hline HDL Cholesterol(mg/dL) & $45,33 \pm 9,32$ \\
\hline Triglyceride (mg/dL) & $159,07 \pm 83,67$ \\
\hline Fasting Insulin (ulU/mL) & $9,9 \pm 8,5$ \\
\hline
\end{tabular}

Table 1. Correlation between visfatin, hs-CRP and HOMA-IR in the obese subject.

\begin{tabular}{llll}
\hline Variable & visfatin $(\mathrm{n}: 76)$ & Homa-IR(n:74) & hs-CRP(n:76) \\
\cline { 2 - 4 } & \multicolumn{1}{c}{$\mathrm{r}$} & $\mathrm{r}$ & $\mathrm{r}$ \\
\hline Waist Circumference $(\mathrm{cm})$ & $-0,046$ & $0,3400,099$ & \\
Fasting Plasma Glucose $(\mathrm{mg} / \mathrm{dL})$ & $-0,043$ & $0,555\left(^{\star \star}\right)$ & $-0,009$ \\
HDL-Cholesterol $(\mathrm{mg} / \mathrm{dL})$ & $0,416\left(^{\star \star}\right)$ & 0,214 & 0,138 \\
Triglyceride $(\mathrm{mg} / \mathrm{dL})$ & $0,260\left(^{*}\right)$ & 0,178 & $-0,019$ \\
HOMA- IR & 0,07 & 0,052 & \\
hs-CRP $(\mathrm{mg} / \mathrm{L})$ & 0,190 & & 0,052 \\
Visfatin $(\mathrm{ng} / \mathrm{mL})$ & & $\mathrm{r}: 0,07$ & $\mathrm{r}: 0,190$ \\
\hline
\end{tabular}




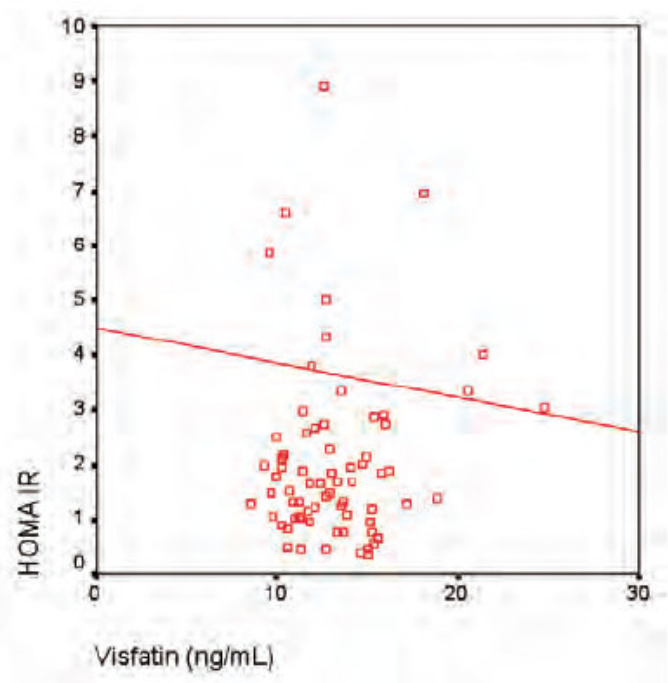

Fig 1. Relationship between visfatin and HOMA-IR

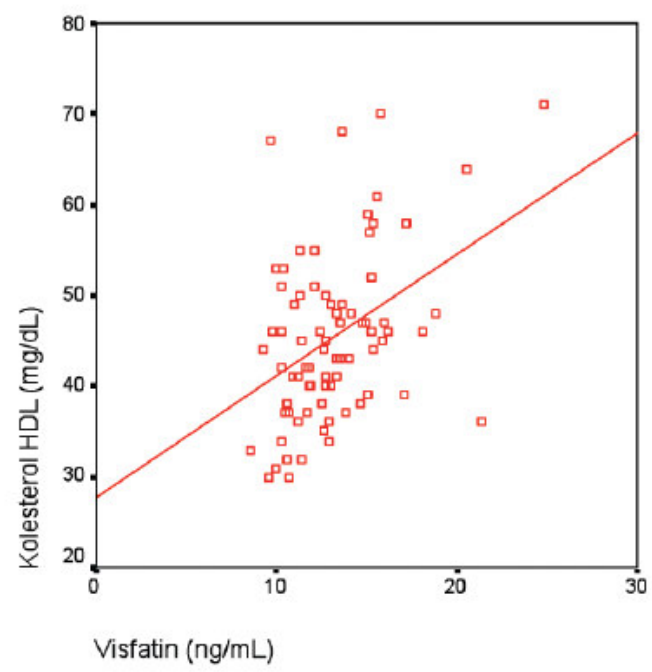

Fig 3. Relationship between visfatin and HDL-Cholesterol

\section{Discussion}

There was no correlation found between visfatin, insulin resistance and inflammation. But suprisingly, we found a strong positive correlation between visfatin and HDL cholesterol concentration.

There were controversial results concerning the association of visfatin with obesity. Studies by LopesBermejo et al. (10), Bailey et al. (11), found the correlation between visfatin and insulin resistance and Markiewics et al. (12), in obese women. But Berndt et al. (13), Sonmez et al. (14) failed to found any correlation between plasma

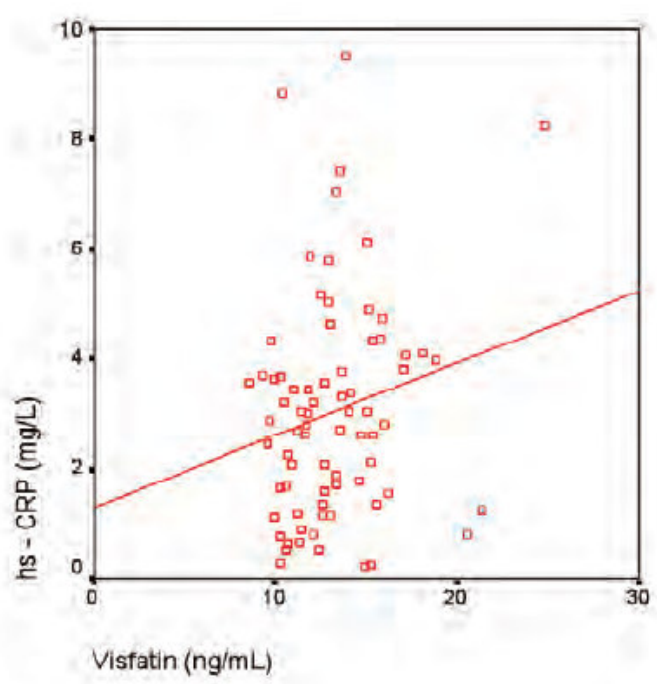

Fig 2. Relationship between visfatin and hsCRP

visfatin with parameters of metabolic syndrome and insulin resistance. Oki et al., 2007 (15) found that serum visfatin levels were positively correlated with the serum levels of iL-6 and slightly related with serum levels of CRP, but not with HOMA-IR in Japanese Americans and Pagano et al. (16) , found no correlation between mRNA visfatin with HOMA-IR.

Differences in the qualitative and quantitative detection of visfatin by immunoassays may explain the conflicting result with respect to putative relation of circulating visfatin to human obesity or insulin resistance. Korner et al. (8), did not find any correlation between visfatin serum levels with visfatin mRNA expression in subcutaneous or visceral fat and with glucose levels.

Visfatin is also known as NAMPT (nicotinamide phosporibosyltranferase) and plays an important role in NAD (nicotinamide adenine dinucleotida) biosynthesis pathway (17). In this study PBEF / NAMPT/ Visfatin had no association with insulin resistance and inflammation, but correlated positively with HDL cholesterol and negatively with triglyceride levels. The same result was found in Indian immigrants (Smithh et al (18), Caucasian subjects (Wang et al.,(19)) and adolescents (Chen et al. (20)). Circulating $\mathrm{PBEF} / \mathrm{NAMPT}$ / visfatin level is an indicator of beneficial lipid profile. The relation to lipid metabolism does not depend on visceral obesity and insulin resistance, but may be linked to its enzymatic function in NAD metabolism. Therefore, we conclude that it is the NAD biosynthetic activity of NAMPT, not the insulin-mimetic activity of visfatin, that is physiologically important for the regulation of lipid metabolism. 


\section{Conclusions}

Circulating levels of visfatin, also known as NAMPT, are correlated with HDL cholesterol concentration. This was with lipid metabolism did not depend on visceral obesity and insulin resistance, but might be linked to visfatin enzymatic function in NAD metabolism .

Further studies are needed to elucidate the mechanisms of visfatin as a novel link with lipid metabolisms.

\section{Acknowledgements:}

The authors thanks the Prodia foundation of Research and Training for their invaluable support in this study.

\section{References:}

1. Wellen, K. E., Hotamisligil, G.S.. Obesity- Induced Inflammatory Changes in Adipose Tissue. J. Clin. Invest 2003; 112: 1785-1788.

2. Varma, V., Borengasser, Y. A., Rasouli, N.,et al, .Human Visfatin Expression: Relationship to Insulin Sensitivity, Intramyocellular Lipids, and Inflammation. J Clin Endocrinol Metab 2007; 92: 666-672

3. Rana, J. S., Nieuwdorp, M.,Jukema, J. W., Kestelein, J.J.P.Cardiovascular Metabolic Syndrome- an Interplay of Obesity, Inflammation, Diabetes and Coronary Heart Disease. Diabetes Obes Metab 2007; $9: 218-232$

4. Eckell, R. H, Grundy, S. M., Zimmet, P. Z. The Metabolic Syndrome. Lancet 2005; 365: 1415-28.

5. Trayhurn, P., Wood, I. S. Adipokines : Inflammation and The Pleiotropic Role of White Adipose Tissue. Brit J Nutr 2004; 92, 345-355

6. Tanaka, M., Nozaki, M., Fukuhara, A., Segawa, K., Aoki N., Matsuda, M., Kumoro R., Shimomura L.. Visfatin is Released From 3T3-L1 Adipocytes Via A non Classical Pathway. Elsevier. 2007. doi : 10.106

7. Fukuhara, A., Matsuda, M., Nishizawa, M.,et al. Visfatin: A Protein Secreted by Visceral Fat That Mimics the Effects of Insulin. Science. 2005; 307, 426. DOI : 10.1126..

8. Korner, A., Garten, A., Bluher, M., et al. Molecular Characteristic of Serum Visfatin and Differential Detection by Immunoassay. J Clin Endrocinol Metab 2007. 92 (12) : 4783-4791
9. Murphy, K. G., Bloom, S. R. Are All Fats Created Equal. Nat Med 2006; $12: 1$

10. Bermejo, L.A., Julia, B.C., Balsells, F.M., et al. serum Visfatin Increased With Progressive $\beta$ Cell Deterioraton. Diabetes 2006; 55: 2871-2875

11. Bailey, D.S., Osti, L.C.J., Lepage, P., et al Common Polymorphisms in The Promoter of the Visfatin Gene (PBEF1) Influence Plasma Insulin Levels in a French-Canadian Population. Diabetes 2006; 55 : 2896-2902.

12. Markiewicz, Z.B., Glinianowics, O.M., Janowska, et al. Serum Concentration of Visfatin in Obese Women.J.Metabol 2006; doi : 10.1016..

13. Berndt, J., Kloting, N., Kralisch, S.,et al. Plasma Visfatin Concentration and Fat Depot- Specific mRNA Expression in Human. Diabetes 2005; 54: 29112916.

14. Sun, G., Bishop, J., Khalili, S.,et al. Serum visfatin Concentrations are Positively Correlated With Serum Triacylgliserol and Down Regulated by Overfeeding in Healthy Young Men. Am J Clin Nutr 2007; 85 : 399-404.

15. Oki, K., Yamane, K., Kamei, N., Nojima, H., Kohno, N. Circulating Visfatin Level Is Correlated With Inflammation, But Not With Insulin Resistance. J Clin Endocrinol Metab 2007; Doi : 10.1111/j.13652265.

16. Pagano, C., Pilon, C., Olivieri, M., Mason, P., et al. Reduced Plasma Visfatin/ Pre-B Cell ColonyEnhancing Factor in Obesity is Not Related to Insulin Resistance in humans. J Clin Endocrinol Metab. 2006;91:3165-7

17. Revollo, R.J., Korner, A., Millis, F.K.,et al. Visfatin Regulates Insulin Secretion in Beta Cells as a Systemic NAD Biosynthesis Enzyme. Cell Metab 2007; $6:$ 363-375.

18. Smith, J., Amri, A.M., Sniderman, A., Cianflone, K. VIsfatin Concetrastion in Asian Indians is Correlated with High Density Lipoprotein Cholesterol and Apolipoprotein A1. Clin Endocrinology 2006; 65 : 667-672.

19. Wang, P. Greevenbroek, V. J.M.M., Bouwman, et al. The Circulating PBEF/ NAMPT/Visfatin level is associated with a Beneficial Blood Lipid Profile. Eur J Physiol 2007; 454:971-976.

20. Chen, P. M., Chung, M.F., Chang, M. D.,et al. Elevated Plasma Level of Visfatin / Pre- $\beta$ Cell ColonyEnhancing Factor in Patients with Type 2 Diabetes Mellitus. Clin Endocrinol Metab 2006; 91 : 295299. 